\title{
Analysis of the Mycobacterium ulcerans genome sequence reveals new loci for variable number tandem repeats (VNTR) typing
}

\author{
Markus Hilty, ${ }^{1} \dagger$ Michael Käser, ${ }^{1}$ Jakob Zinsstag, ${ }^{1}$ Tim Stinear $^{2}$ \\ and Gerd Pluschke ${ }^{1}$ \\ ${ }^{1}$ Swiss Tropical Institute, 4002 Basel, Switzerland \\ ${ }^{2}$ Department of Microbiology, Monash University, Clayton, Victoria, Australia
}

Markus Hilty

m.hilty@imperial.ac.uk

Received 24 November 2006

Revised 16 January 2007

Accepted 17 January 2007
Screening of the genome sequence of the Mycobacterium ulcerans strain Agy99 from Ghana with tandem repeats finder software revealed 34 novel non-degenerate tandem repeats containing loci suitable for variable number tandem repeats (VNTR) typing. All loci revealed polymorphism within M. ulcerans isolates of geographically diverse origins. The results confirm the evolutionary scenario suggested by multi-locus sequence typing in which a progenitor of all $M$. ulcerans lineages emerged from the environmental species Mycobacterium marinum and subsequently diverged into several geographical lineages. For further attempts to develop a VNTR-based genetic fingerprinting tool for $M$. ulcerans, it is suggested that the focus should rather be on M. marinum than on the African M. ulcerans Agy99 genome sequence as a starting point.

\section{INTRODUCTION}

Mycobacterium ulcerans, a slow-growing environmental mycobacterium (Yeboah-Manu et al., 2004), causes Buruli ulcer, which is, after tuberculosis and leprosy, the third most important mycobacterial disease worldwide (Sizaire et al., 2006; van der Werf et al., 2003). Buruli ulcer is mainly prevalent in West Africa but cases have also been reported from various other tropical and sub-tropical areas of the world (Asiedu et al., 2000; Johnson et al., 2005). Genetic analysis indicates the emergence of $M$. ulcerans from $M$. marinum, a fish pathogen which can also cause disease in humans (Boddinghaus et al., 1990; Tonjum et al., 1998). There is a strong association between the occurrence of $M$. ulcerans infection in humans and slow-flowing or stagnant water bodies (Marsollier et al., 2007; Portaels et al., 1999; Roberts \& Hirst, 1997; Ross et al., 1997; Stinear et al., 2000a). Aquatic snails (Marsollier et al., 2004), fish (Eddyani et al., 2004) and water bugs (Marsollier et al., 2002) are currently being discussed as passive and active hosts, respectively. However, the exact mode of transmission of Buruli ulcer has remained a mystery, partly because no genetic fingerprinting tool is available for micro-epidemiological analysis of infection chains.

Variable number tandem repeats (VNTR) typing is a promising typing tool for M. ulcerans, because analysis based on few loci already has a higher discriminatory power

tPresent address: Imperial College, National Heart and Lung Institute, Dovehouse Street, London SW3 6LY, UK.

Abbreviation: VNTR, variable number tandem repeats. than other standard molecular typing methods (Ablordey et al., 2005a, b; Hilty et al., 2006; Stragier et al., 2005, 2006). VNTR typing thus revealed for the first time genetic diversity within isolates from African countries (Hilty et al., 2006; Stragier et al., 2006). So far, two approaches have been used to identify loci suitable for VNTR typing of M. ulcerans. These are the screening of the Mycobacterium marinum genome sequence for tandem repeats containing loci with tandem repeats finder software (Ablordey et al., 2005b; Hilty et al., 2006), and BLAST searches for homologues of M. tuberculosis mycobacterial interspersed repetitive units in $M$. ulcerans (Stragier et al., 2005). Here, we have searched for the first time the completely assembled $M$. ulcerans genome sequence for tandem repeats containing loci and have evaluated the potential of the identified loci for VNTRbased typing.

\section{METHODS}

Identification of the tandem-repeat-containing loci. Tandem repeats finder software (http://tandem.bu.edu/trf/trf.html) was used to screen the M. ulcerans Agy99 sequence database and to identify tandem-repeat-containing loci. Selection criteria were a repeat size of $>20 \mathrm{bp}$, a perfect $(100 \%)$ sequence identity of the repeats and an entropy of repeat sequences $>1.70$ within the total entropy range of 0-2 to allow for easy determination of the number of repeats by agarose gel electrophoresis and to exclude very similar (e.g. GC-rich) sequences which do not represent tandem repeats.

Primer design. Specific primers targeting repeat-flanking genomic sequences of $M$. ulcerans Agy 99 were designed using Frodo Primer 3 software (http://frodo.wi.mit.edu/). According to sequence information from the M. marinum genome database (www.sanger.ac.uk/ projects/M_marinum), the $M$. marinum sequences corresponding to 
Table 1. Newly identified VNTR loci and primers used for VNTR typing

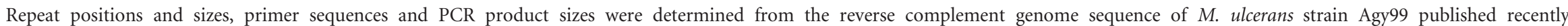
(Stinear et al., 2007). Primers which had single nucleotide differences in the M. marinum target sequence are indicated in bold (primer no.)

\begin{tabular}{|c|c|c|c|c|c|}
\hline $\begin{array}{l}\text { VNTR } \\
\text { locus no. }\end{array}$ & $\begin{array}{c}\text { Position in } \\
\text { Agy99 genome }\end{array}$ & Repeat sequence (repeat size) & $\begin{array}{c}\text { Size of PCR } \\
\text { product (Agy99) }\end{array}$ & Primer no. & Primer sequence \\
\hline \multirow[t]{2}{*}{1} & 75046 & AGAAGCCGAGCTGACGCGACGCGGCCAACCCTTCAAA (37) & 232 & 1579 & CATAGCCACGTGACCAATGT \\
\hline & & & & 1580 & GTGAGCAGGGCATCTACCAG \\
\hline \multirow[t]{2}{*}{2} & 260371 & ATGCCACGGTGGGCAATCCCTACCCCG (27) & 238 & 1671 & TGGTGTGGTTGCGTCTTCT \\
\hline & & & & 1672 & GGACAAAGACGCGAGAAAGT \\
\hline \multirow[t]{2}{*}{3} & 624989 & CTGTTCAAGGGATTCAATGTGGGCCTCG (28) & 236 & 1623 & AAGATCATTCTGCCGCTCAT \\
\hline & & & & 1624 & CACAACCGAAGATCAACACG \\
\hline \multirow[t]{2}{*}{4} & 688860 & AGTTCCTCTCGCCAGGCATGGATGC (25) & $185^{\star}$ & 1673 & CGTTGTGACACGTCATCCAT \\
\hline & & & & 1674 & GTTCCGGTCTGCGATACTGT \\
\hline \multirow[t]{2}{*}{5} & 719016 & TCTTCTCCTTCGACTTGGGCAGGCGTC (27) & 362 & 1625 & CAACCTCGTCGAGATCAACC \\
\hline & & & & 1626 & AAGGTGACGCAAGCAATGTT \\
\hline \multirow[t]{5}{*}{6} & 759207 & TGCCGAAATCAGCCCGACGGCGCAGGCCCAGCTGGCGGC- & 388 & 1581 & GCGGGGTATCTGACCAATAG \\
\hline & & CGTCGCCGGCGGCCAAGATCCGGTGACACTGAACCTCGA- & & & \\
\hline & & TGGGTTGGGCCGCTACCGGGTGGTGTCCGCCCTGAGCCG- & & & \\
\hline & & CCGCGGTGACGAACTCATCGTCACCGGCCTTTCGA (152) & & & \\
\hline & & & & 1582 & GTGACGATGCCGAAAATGAT \\
\hline \multirow[t]{2}{*}{7} & 898478 & TCCTTTTAGAACCCTCCAAGCGCG (24) & 232 & 1675 & CTTTCTCCATGCGGGAGTT \\
\hline & & & & 1676 & TCGTTGCTGTCACCAGACTC \\
\hline \multirow[t]{2}{*}{8} & 1023923 & CGCCCGCTGGTGAGGCCGTTCCGGACGAAC (30) & 304 & 1583 & CTTCCCATCAATGGTTTTGG \\
\hline & & & & 1584 & GCCCGGTGAACCAACTACTA \\
\hline \multirow[t]{2}{*}{9} & 1440525 & GCCACCCTACGGACCAATTAC (21) & 160 & 1679 & CGCACTGGTGAGTCTGGATA \\
\hline & & & & 1680 & GGCATCCAACTTGACTGACA \\
\hline \multirow[t]{2}{*}{10} & 1563743 & CGAGCTGTGTTCTGCTGGTGCGCCAT (26) & 278 & 1627 & CATCGCCACAAGTGTTTTCA \\
\hline & & & & 1628 & TTCGGTTCCTTTCACATGGT \\
\hline \multirow[t]{2}{*}{11} & 1773797 & CACССТTTGCCCAACACTTGC (21) & 194 & 1681 & AAACGACATCGACCAGACG \\
\hline & & & & 1682 & CCGAGTCGAAGTTGGTATGC \\
\hline \multirow[t]{2}{*}{12} & 1973523 & GTCGGCACCGATGAGGTAATCG (22) & 189 & 1683 & CCGCATACGATCTGTTCAGT \\
\hline & & & & 1684 & ACCGAAAGTCCAGGGTTCTT \\
\hline \multirow[t]{2}{*}{13} & 2018640 & GCCACATATGGCAACCCCTTGGGGATAC (28) & $317^{\star}$ & 1591 & AACTCCGGGATTGGTTCTTT \\
\hline & & & & 1592 & AACGTCGTCTGTTTCGGTTC \\
\hline \multirow[t]{2}{*}{14} & 2314530 & GAATGCCGTGTCTTCACAAACTGCCG (26) & 325 & 1629 & GACCGTAAACCGACGAAAAA \\
\hline & & & & 1630 & CACTAATATGCGGTGCGTTC \\
\hline \multirow[t]{2}{*}{15} & 2475473 & CGACACCCGGTATTCCCGCATAT (23) & 228 & 1685 & CCGTAGATGGTCTGGATGC \\
\hline & & & & 1686 & CCCGAACCTATCTCGATGAC \\
\hline \multirow[t]{2}{*}{16} & 2549331 & CGCATTGCGAAGTGGCGGACG (21) & 352 & 1631 & ATGCAACCACTTGCCCATC \\
\hline & & & & 1632 & AGCAGTTCTTCGGTGACGAC \\
\hline
\end{tabular}


Table 1. cont.

\begin{tabular}{|c|c|c|c|c|c|}
\hline $\begin{array}{l}\text { VNTR } \\
\text { locus no. }\end{array}$ & $\begin{array}{c}\text { Position in } \\
\text { Agy99 genome }\end{array}$ & Repeat sequence (repeat size) & $\begin{array}{c}\text { Size of PCR } \\
\text { product }(A g y 99)\end{array}$ & Primer no. & Primer sequence \\
\hline \multirow[t]{2}{*}{17} & 2847974 & AGACTCCGGAGTTCATGGCCATTCGTC (27) & 304 & 1585 & GTTATCACCCGATTGCCAGT \\
\hline & & & & 1586 & AAACAACGACTTTCGCGTCT \\
\hline \multirow[t]{2}{*}{18} & 3474730 & ACCTCGGCGGTACCCGCGAACAG (28) & 250 & 1587 & AGGTCGACTTCGTGCTCAAC \\
\hline & & & & 1588 & ATGGGCGTAGGTCCAAAAT \\
\hline \multirow[t]{2}{*}{19} & 3709194 & TGATCTCGCCTTCCTCGTTACCCACCGG (28) & 224 & 1589 & AGGATCGCCTTCTTGTTTCC \\
\hline & & & & 1590 & TGCAGTGCATCGTTCATACC \\
\hline \multirow[t]{2}{*}{20} & 3802904 & ACCGATCTATTGGCCGCAAAG (21) & 250 & 1687 & TGAGTTGGCCCAGTCGAG \\
\hline & & & & 1688 & GACAAGCCGGCCAACATC \\
\hline \multirow[t]{2}{*}{21} & 3913042 & ACCATTGCCGAGCTGGTGAAT (21) & 279 & 1635 & GTCACCGTCAACATCACGTC \\
\hline & & & & 1636 & GGTCAGCACGTTTTCATGC \\
\hline \multirow[t]{2}{*}{22} & 4062301 & ATGAATTCTTGGCAGATTCTT (21) & 313 & 1637 & GTATGCGCGACTGATCCAC \\
\hline & & & & 1638 & CCGTTTGGCTATCCGATAAA \\
\hline \multirow[t]{2}{*}{23} & 4110344 & AAATCGTCACGCCGGGAAGTT (21) & 182 & 1691 & GGACCGACTGTTGATGGTTT \\
\hline & & & & 1692 & TGGAGTCCAGGAAGAACTCG \\
\hline \multirow[t]{2}{*}{24} & 4145439 & CAGTCATCGGAATTCCCCCAATC (23) & 315 & 1639 & GAAGCCCATCGTGTCTGC \\
\hline & & & & 1640 & CTTACCTGGGCGATGAGTGT \\
\hline \multirow[t]{2}{*}{25} & 4251686 & AACCTGCCCTGGTCGTCGGAGTT (23) & 355 & 1641 & CAGCAGGTCCGCTATGAAGT \\
\hline & & & & 1642 & GTCGTTGAAGGGGTGACACT \\
\hline \multirow[t]{2}{*}{26} & 4450491 & CGAGCGCAGGTGCACATGGTG (21) & $193 \dagger$ & 1693 & TTGCCGAAATTTCTCTTTGG \\
\hline & & & & 1694 & AGTCGGTCAGGGGTTGGT \\
\hline \multirow[t]{2}{*}{27} & 4559395 & TTCAGTCAGGGGCTGAGCAAC (21) & 150 & 1695 & TGAGCCGATAGTGCAAAGTG \\
\hline & & & & 1696 & TCGGTTTGGATGAATTGGTT \\
\hline \multirow[t]{2}{*}{28} & 4596959 & CGGCTTTCAAATCGTTTTGTCGGTCGAC (28) & 324 & 1593 & TCTACCCCAACACCGAGAAC \\
\hline & & & & 1594 & ATCCAAAATGGACACCGAAG \\
\hline \multirow[t]{2}{*}{29} & 4837553 & CTATGGCGACACCTACACCGA (21) & 231 & 1697 & TGATTCCCAAGACGAGAACC \\
\hline & & & & 1698 & CCGTTCGTCGTCAAGTTGTA \\
\hline \multirow[t]{2}{*}{30} & 4964512 & CGCCCAGAACAGCCGTCATAC (21) & 211 & 1699 & ACTTCACCAACGGCTTTCAC \\
\hline & & & & 1700 & ACCAACTCCAAGGTCACCAG \\
\hline \multirow[t]{2}{*}{31} & 5006187 & CGGGTCGCCGGCCTCACATCT (21) & 310 & 1643 & GTCAGGGCTTTGATCTCACC \\
\hline & & & & 1644 & CGCTGCACGTCAAAACTAAG \\
\hline \multirow[t]{2}{*}{32} & 5135404 & GCTCCGCTGCCTAGGTTCGAC (21) & 344 & 1645 & CCAGTTGCTGCAGAAGTTCA \\
\hline & & & & 1646 & CGAAGTGAAAGGTCGTTTGG \\
\hline \multirow[t]{2}{*}{33} & 5210577 & GGTTGTCCAGCCGCGGCGCGCTGA (24) & 240 & 1701 & GTGTTCAGCAGGTTGGACTG \\
\hline & & & & 1702 & TCTGATGACCCATGACCTGA \\
\hline \multirow[t]{2}{*}{34} & 5323208 & ATCAACTATCTGTCGCAGACCGGTGCGTT (29) & 301 & 1597 & TGCTGCTGGATTGTTTTCG \\
\hline & & & & 1598 & CATGATGCCAAGCAGCAC \\
\hline
\end{tabular}

*French Guyana isolate did not amplify. 
these primers were $100 \%$ identical, except for four primers, 1676, 1682, 1684 and 1692, which contained single nucleotide mismatches (Table 1).

PCR analysis and copy number designation. A panel consisting of $11 \mathrm{M}$. ulcerans clinical isolates of human origin from diverse geographical areas was used to assess the polymorphism of the VNTR loci. PCR for tandem-repeat-containing loci (Table 1) was performed as described by Hilty et al. (2006). Briefly, PCR mixtures (final volume, $20 \mu \mathrm{l}$ ) contained $2 \mu \mathrm{l}$ Taq PCR buffer, deoxynucleoside triphosphates $(0.2 \mathrm{mM}$ each), $1 \mathrm{U}$ AmpliTaq DNA polymerase (Perkin-Elmer Applied Biosystems), each primer at a concentration of $0.5 \mu \mathrm{M}$ and mycobacterial DNA. PCR was performed for 40 cycles of $0.5 \mathrm{~min}$ at $94^{\circ} \mathrm{C}, 0.5 \mathrm{~min}$ at $65^{\circ} \mathrm{C}$ and $1 \mathrm{~min}$ at $72^{\circ} \mathrm{C}$. The reactions were terminated by incubation for $10 \mathrm{~min}$ at $72^{\circ} \mathrm{C}$.

PCR fragments were analysed by agarose gel electrophoresis using $2 \%$ NuSieve agarose, and sizes of the amplicons were estimated by comparison with Size Marker VIII (Roche), compared with the product size of Agy 99 and copy number designated.

\section{RESULTS AND DISCUSSION}

Screening of the M. ulcerans strain Agy99 genome sequence from Ghana with tandem repeats finder software identified 45 perfect tandem-repeat-containing loci which have not been described in previous studies (Ablordey et al., 2005a, b; Hilty et al., 2006; Stragier et al., 2005, 2006). Eleven out of 45 loci were excluded from further analysis for various reasons, including the presence of insertion sequence elements. For the remaining 34 loci, primers were designed (Table 1) and PCR amplification of a panel of 11 wellcharacterized M. ulcerans clinical isolates of diverse geographical origins performed. As it was our primary objective to develop a genetic fingerprinting method for African $M$. ulcerans isolates, more strains from Africa (6) than from other continents were analysed.

All 34 loci showed polymorphisms within the 11 isolates tested, but only alleles with one and with two repeats were found. Based on the typing results, the new VNTR loci were classified into four different subgroups, designated i-iv (Table 2). While subgroups i and ii comprised a multitude of loci (17 and 15, respectively), subgroups iii and iv were each represented by only one locus. For all 34 new VNTR loci, the $M$. marinum genome sequencing strain and the $M$. ulcerans isolates from Japan and French Guyana had only one repeat (genotype A; Table 2). In contrast, all six African M. ulcerans isolates analysed plus strain Agy99 from Ghana had two repeats in all 34 loci (VNTR genotype E; Table 2), indicating that they have further diverged from $M$. marinum than the other tested geographical lineages of M. ulcerans. The isolates from Australia, Malaysia and Papua New Guinea had one repeat in all 17 subgroup i loci and two repeats in all 15 subgroup ii loci, thus sharing a comparable number of alleles with both the M. marinum-associated VNTR genotype A and the African M. ulcerans-associated genotype E. They could be further subdivided into three VNTR genotypes (designated B-D) by differences in the two VNTR loci 15 and 24 constituting subgroups iii and iv (Table 2). These results indicate an evolutionary scenario in which a progenitor of all $M$. ulcerans lineages emerged from M. marinum, subsequently diverging into several geographical lineages. The African lineage may have evolved from an ancestral lineage from the Western Pacific region (Australia, Malaysia and Papua New Guinea) which may itself have a progenitor in Asia. These results may confirm the evolutionary scenario suggested by multi-locus sequence typing (Stinear et al., 2000b).

In this study, our selection criteria for finding tandemrepeat-containing loci were highly stringent and it should be possible to identify more VNTR loci, such as degeneraterepeat-containing loci and loci with repeat sizes of $<20 \mathrm{bp}$. While for all 34 new loci identified here by screening of the M. ulcerans Agy99 genome sequence only alleles with one and with two repeats were found, Ablordey et al. (2005b)

Table 2. VNTR genotypes defined by the 34 new VNTR loci

\begin{tabular}{|c|c|c|c|c|c|}
\hline \multirow{2}{*}{$\begin{array}{l}\text { VNTR } \\
\text { genotype }\end{array}$} & \multirow{2}{*}{$\begin{array}{c}\text { Origin of } \\
\text { strains }^{*}\end{array}$} & \multicolumn{3}{|c|}{ No. of tandem repeats in the subgroups of new } & \multirow{2}{*}{$\begin{array}{l}\text { VNTR loci } \dagger \\
\text { iv (1 locus) }\end{array}$} \\
\hline & & i (17 loci) & ii (15 loci) & iii (1 locus) & \\
\hline A & $\begin{array}{l}\text { Japan, French Guyana, } \\
\text { M. marinum }\end{array}$ & 1 & 1 & 1 & 1 \\
\hline B & Australia & 1 & 2 & 1 & 1 \\
\hline $\mathrm{C}$ & Malaysia & 1 & 2 & 2 & 1 \\
\hline $\mathrm{D}$ & Papua New Guinea & 1 & 2 & 2 & 2 \\
\hline E & Africa & 2 & 2 & 2 & 2 \\
\hline
\end{tabular}

${ }^{\star} J a p a n$, ITM-8756; French Guyana, ITM-7922; M. marinum, derived from the M. marinum genome sequence (www.sanger.ac.uk/projects/M_marinum); Australia, ITM-884; Malaysia, ITM-941328; Papua New Guinea, ITM-9357. African isolates: Ghana, ITM-970321 and ITM-970359; Benin, ITM-940512 and ITM-940886; Ivory Coast, ITM-940662; Angola, ITM-960658; and Agy99 (derived from http://genopole. pasteur.fr/Mulc/BuruList.html).

†Subgroup I, VNTR 1, 6, 7, 8, 9, 11, 12, 14, 18, 19, 21, 23, 26, 29, 30, 31 and 34; subgroup ii, 2, 3, 4, 5, 10, 13, 16, 17, 20, 22, 25, 27, 28, 32 and 33; subgroup iii, VNTR 15; subgroup iv, VNTR 24. 
also described alleles with more than two repeats for $6 / 9$ VNTR loci identified by screening the M. marinum genome. M. marinum genome-assisted identification of new VNTRs has led to the first demonstration of genetic diversity in the same panel of African M. ulcerans isolates as used in this study (Hilty et al., 2006). In contrast, the strain Agy99 genome-based approach used here did not identify another VNTR locus that differentiates African isolates. Therefore, attempts to refine VNTR-based genetic fingerprinting for micro-epidemiological analysis of M. ulcerans should rather focus on the M. marinum than on the M. ulcerans Agy99 genome sequence as a starting point.

\section{ACKNOWLEDGEMENTS}

We thank Professor F. Portaels of the Institute of Tropical Medicine (ITM) for providing $M$. ulcerans strains and the head of the $M$. marinum genome sequencing project for permission to use the sequence data. M. H. and M. K. were funded by the National Centre of Competence in Research (NCCR) North-South IP-4 and Deutsche Forschungsgemeinschaft grant KA 1842/1-1, respectively.

\section{REFERENCES}

Ablordey, A., Hilty, M., Stragier, P., Swings, J. \& Portaels, F. (2005a). Comparative nucleotide sequence analysis of polymorphic variablenumber tandem-repeat loci in Mycobacterium ulcerans. J Clin Microbiol 43, 5281-5284.

Ablordey, A., Swings, J., Hubans, C., Chemlal, K., Locht, C., Portaels, F. \& Supply, P. (2005b). Multilocus variable-number tandem repeat typing of Mycobacterium ulcerans. J Clin Microbiol 43, 1546-1551.

Asiedu, K., Scherpbier, R. \& Raviglione, M. (2000). Buruli Ulcer Mycobacterium ulcerans Infection. WHO Document, WHO/CDS/ CPE/BGUI/2000.1.

Boddinghaus, B., Rogall, T., Flohr, T., Blocker, H. \& Bottger, E. C. (1990). Detection and identification of Mycobacteria by amplification of ribosomal-RNA. J Clin Microbiol 28, 1751-1759.

Eddyani, M., Ofori-Adjei, D., Teugels, G., De Weirdt, D., Boakye, D., Meyers, W. M. \& Portaels, F. (2004). Potential role for fish in transmission of Mycobacterium ulcerans disease (Buruli ulcer): an environmental study. Appl Environ Microbiol 70, 5679-5681.

Hilty, M., Yeboah-Manu, D., Boakye, D., Mensah-Quainoo, E., Rondini, S., Schelling, E., Ofori-Adjei, D., Portaels, F., Zinsstag, J. \& Pluschke, G. (2006). Genetic diversity in Mycobacterium ulcerans isolates from Ghana revealed by a newly identified locus containing a variable number of tandem repeats. J Bacteriol 188, 1462-1465.

Johnson, P. D., Stinear, T., Small, P. L., Pluschke, G., Merritt, R. W., Portaels, F., Huygen, K., Hayman, J. A. \& Asiedu, K. (2005). Buruli ulcer (M. ulcerans infection): new insights, new hope for disease control. PLoS Med 2, e108.

Marsollier, L., Robert, R., Aubry, J., Saint Andre, J. P., Kouakou, H., Legras, P., Manceau, A. L., Mahaza, C. \& Carbonnelle, B. (2002). Aquatic insects as a vector for Mycobacterium ulcerans. Appl Environ Microbiol 68, 4623-4628.
Marsollier, L., Severin, T., Aubry, J., Merritt, R. W., Saint Andre, J. P., Legras, P., Manceau, A. L., Chauty, A., Carbonnelle, B. \& Cole, S. T. (2004). Aquatic snails, passive hosts of Mycobacterium ulcerans. Appl Environ Microbiol 70, 6296-6298.

Marsollier, L., Andre, J. P., Frigui, W., Reysset, G., Milon, G., Carbonnelle, B., Aubry, J. \& Cole, S. T. (2007). Early trafficking events of Mycobacterium ulcerans within Naucoris cimicoides. Cell Microbiol 9, 347-355.

Portaels, F., Elsen, P., Guimaraes-Peres, A., Fonteyne, P. A. \& Meyers, W. M. (1999). Insects in the transmission of Mycobacterium ulcerans infection. Lancet 353, 986.

Roberts, B. \& Hirst, R. (1997). Immunomagnetic separation and PCR for detection of Mycobacterium ulcerans. J Clin Microbiol 35, 2709-2711.

Ross, B. C., Marino, L., Oppedisano, F., Edwards, R., RobinsBrowne, R. M. \& Johnson, P. D. (1997). Development of a PCR assay for rapid diagnosis of Mycobacterium ulcerans infection. J Clin Microbiol 35, 1696-1700.

Sizaire, V., Nackers, F., Comte, E. \& Portaels, F. (2006). Mycobacterium ulcerans infection: control, diagnosis, and treatment. Lancet Infect Dis 6, 288-296.

Stinear, T., Davies, J. K., Jenkin, G. A., Hayman, J. A., Oppedisano, F. \& Johnson, P. D. (2000a). Identification of Mycobacterium ulcerans in the environment from regions in Southeast Australia in which it is endemic with sequence capture-PCR. Appl Environ Microbiol 66, 3206-3213.

Stinear, T. P., Jenkin, G. A., Johnson, P. D. R. \& Davies, J. K. (2000b). Comparative genetic analysis of Mycobacterium ulcerans and $\mathrm{Myco-}$ bacterium marinum reveals evidence of recent divergence. J Bacteriol 182, 6322-6330.

Stinear, T. P., Seemann, T., Pidot, S., Frigui, W., Reysset, G., Garnier, T., Meurice, G., Simon, D., Bouchier, C. \& other authors (2007). Reductive evolution and niche adaptation inferred from the genome of Mycobacterium ulcerans, the causative agent of Buruli ulcer. Genome Res 17, 192-200.

Stragier, P., Ablordey, A., Meyers, W. M. \& Portaels, F. (2005). Genotyping Mycobacterium ulcerans and Mycobacterium marinum by using mycobacterial interspersed repetitive units. J Bacteriol 187, 1639-1647.

Stragier, P., Ablordey, A., Bayonne, L. M., Lugor, Y. L., Sindani, I. S., Suykerbuyk, P., Wabinga, H., Meyers, W. M. \& Portaels, F. (2006). Heterogeneity among Mycobacterium ulcerans isolates from Africa. Emerg Infect Dis 12, 844-847.

Tonjum, T., Welty, D. B., Jantzen, E. \& Small, P. L. (1998). Differentiation of Mycobacterium ulcerans, M. marinum, and M. haemophilum: mapping of their relationships to $M$. tuberculosis by fatty acid profile analysis, DNA-DNA hybridization, and 16S rRNA gene sequence analysis. J Clin Microbiol 36, 918-925.

van der Werf, T. S., Stinear, T., Stienstra, Y., van der Graaf, W. T. A. \& Small, P. L. (2003). Mycolactones and Mycobacterium ulcerans disease. Lancet 362, 1062-1064.

Yeboah-Manu, D., Bodmer, T., Mensah-Quainoo, E., Owusu, S., Ofori-Adjei, D. \& Pluschke, G. (2004). Evaluation of decontamination methods and growth media for primary isolation of Mycobacterium ulcerans from surgical specimens. J Clin Microbiol 42, 5875-5876.

Edited by: G. S. Besra 